\title{
Efficient Wireless Scheduling with Limited Channel Feedback and Performance Guarantees
}

\author{
Mehmet Karaca, Yunus Sarikaya, Ozgur Ercetin, Tansu Alpcan, Holger Boche
}

\begin{abstract}
It is well known that Max-Weight scheduling provides queue stability whenever this is possible. However, MaxWeight scheduling requires the complete channel state information (CSI) to make the best transmission decision at every time slot. The common assumption in this line of research assumes that the network controller has full CSI at every decision time without taking into account the overhead associated with channel probing. In practice, however, acquiring CSI is not cost-free and requires certain amount of resources. In this work, we design a Scheduling and Dynamic Feedback algorithm, named SDF, by considering the overhead of obtaining the channel state information. We first establish a bound on the achievable rate region of SDF algorithm by proving that SDF supports $1+\epsilon$ fraction of of the full rate region (the rate region when all users are probed) where $\epsilon$ only depends on the expected number of users which are not probed. Then, for homogenous channel, we show that when the number of users in the network is greater than $3, \epsilon>0$, i.e., we guarantee to expand the rate region. We also demonstrate numerically in a realistic simulation setting that this rate region can be achieved by probing only less than $50 \%$ of all channels in a CDMA based cellular network utilizing high data rate protocol under normal channel conditions.
\end{abstract}

\section{INTRODUCTION}

The scheduling is an essential problem for any shared resource. The problem becomes more challenging in a dynamic setting such as wireless networks where the channel capacity is time varying due to multiple superimposed random effects such as mobility and multipath fading. In a queueing system, the most important property of a scheduling algorithm is to support traffic rate as high as possible while keeping the network stable (e.g., the queue sizes remain bounded over time). All stabilizable traffic rates form a region which we call achievable rate region of the network. The seminal work by Tassiulas and Ephremides has shown that Max-Weight algorithm scheduling the user with the highest queue backlog and transmission rate product at every time slot achieves the largest achievable rate region and stabilizes the network [1].

There has been much work in developing scheduling algorithms for wireless systems with various performance metrics that include stability, utility maximization and energy minimization [1], [2], [3]. However, the common assumption in these works is that the exact and complete channel state

Mehmet Karaca, Yunus Sarikaya and Ozgur Ercetin are with the Faculty of Engineering and Natural Sciences, Sabanci University. Email: \{mehmetkrc,sarikaya,oercetin\}@ sabanciuniv.edu.

Tansu Alpcan is with Dept of Electrical and Electronic Engineering, The University of Melbourne. Email: tansualpcan@gmail.com

Holger Boche is with the Lehrstuhl für Theoretische Informationstechnik, Technische Universität München, Germany. Email: boche@tum.de

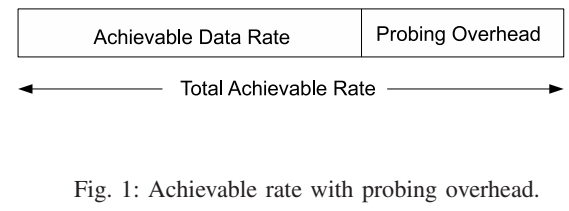

information, (CSI) of all users is available at every time slot. However, in practice acquiring CSI introduces significant overhead to the network, since CSI is obtained either by probing the channel or via feedback from the users. As shown Figure 1, only some portion of total achievable data rate is available when probing cost is taken into account.

As a motivating example, consider CDMA/HDR (High Data Rate) system [4], where the Signal-to-Noise Ratio (SNR) of each link is measured. The value of the SNR is sent back to the base station via the reverse link data rate request channel (DRC). The channel state information is updated every 1.67 ms and sent back as 4 bits. Assume that there are 25 users in a cell, and hence, 100 bits of channel information has to be sent every $1.67 \mathrm{~ms}$. This requires $60 \mathrm{Kbps}$ of channel rate to be dedicated only for channel measurements. The minimum data rate of HDR system is $38.4 \mathrm{Kbps}$ and the average data rate is $308 \mathrm{Kbps}$. Thus, overhead of acquiring CSI is twice the minimum data rate, and is approximately more than $20 \%$ of the average transmission rate. This overhead increases significantly in a multichannel communication system such as LTE. Thus, channel probing must be done efficiently in order to balance the trade-off between being opportunistic (e.g., obtaining useful channel information) and consuming valuable resources.

In this work, we consider in fully connected network (e.g., Cellular network, WLAN) where base station (BS) is transmitting to a fixed number of users. We assume that each user has infinite buffer capacity and data arrives into users' queues according to a stochastic arrival process. We aim to develop a joint scheduling and channel probing algorithm that both stabilizes users' queues with taking into account to the probing cost and is easy to implement.

Our contributions are summarized as follows:

- We first propose a joint scheduling and channel probing algorithm which is easy to implement and low cost. In addition, our algorithm does not require any statistics (i.e., channel or arrival statistics) and can be applied over correlated and even non-stationary channels.

- Technical contributions of our paper are as follows: for 
homogenous channels, we first show that the proposed algorithm can achieve $1+\epsilon$ fraction of the full rate region of the case when all users are probed. Then, we analytically show that $\epsilon>0$ (e.g., we guarantee to increase the rate region) when the number of users is greater than 3 .

- We implement a realistic network setting where we simulate High Data Rate (HDR) protocol in CDMA cellular networks and show by numerical analysis that when our proposed algorithm is used a comparable performance with Max-Weight algorithm with full CSI can be achieved by only probing on the average less than $50 \%$ of users.

\section{RELATED WORKS}

In [5], the authors proposed a joint scheduling and channel probing algorithm stabilizing the network by allowing the base station to probe a subset of channels (or links) at each time slot. However, throughput-optimality of that algorithm can only be shown under certain condition, i.e., when channels are symmetric and subsets of channels are disjoint. In [6], a feedback allocation algorithm was proposed for multi-channel system with limited feedback bandwidth. In other words, only a limited number of users can be probed at a time.

In [7], the authors proposed a scheduling algorithm under imperfect CSI in single-hop networks with i.i.d. channels. However, it was assumed that probing a channel brings a certain amount of energy cost rather than time cost. [8] attempts to learn transmission success probabilities by averaging the previous outcomes by assuming i.i.d. channel processes across time. In [9], the authors proposed to estimates the channel statistics by using some portion of the time slots for observation slot with some probability over i.i.d channels. However, rather than estimation channel states as in [8] and [9], we investigate stability when channel probing brings cost. In [10] and [11], it was assumed that wireless channels evolve as Markov-modulated ON/OFF processes. With this assumption, a exploitation-exploration trade-off was investigated. The performance of [10] and [11] depends on the underlaying stochastic process of the channel evolves according to a fixed stationary process such ergodic Markov chain. In our work, we assume i.i.d channel model which is more realistic for fast fading channels.

Unlike the works above, we assume a more flexible channel probing model where BS dynamically decides the number of probed user at each time slot. In this sense, the most related work is [12], where the optimal feedback- scheduling scheme for a single-channel downlink is derived. Specifically, in [12], it was assumed that a single channel probing requires a certain portion of data transmission (i.e., $\beta$ fraction of data slot). The problem of finding optimal joint algorithm is transformed into an optimal stopping time problem and is solved by Markov Decision Process (MDP) where the channel probabilities are known to the BS. The main drawbacks of [12] are as follows: first, since the authors uses MDP to solve the problem, this formulation is computationally intractable as it involves a high dimensional state. Second, it requires channel distributions to determine probing decision. Third, optimal transmission and probing policy is given under certain channel conditions, i.e., proportional fading. Lastly, [12] does not provide an explicit bound on the achievable rate region.

\section{SySTEM MODEL}

We consider a time slotted $t \in\{0,1,2, \ldots\}$ cellular system with a single base station (BS) transmitting to $N$ users with a fixed power. Let $\mathcal{N}$ denote the set of users in the cell. Wireless channel between the base station and a mobile user is assumed to be independent across users and slots. The gain of the channel is constant over the duration of a time slot but varies between slots. In practical systems (e.g., CDMA/HDR system [4]), transmission rate is determined by a link adaptation algorithm, which selects the highest transmission rate to meet a given allowable target error probability. Only finite number of transmission rates can be supported due to modulation and coding schemes. We assume that each channel has $L$ possible states with corresponding rates $\mathcal{R}=\left\{r_{1}, r_{2}, \ldots, r_{L}\right\}$ listed in descending order, i.e., $r_{k}>r_{l}$ if $l>k$. Note that $r_{k}$, $k \in\{1,2, \ldots, €\}$ only depends on the Signal-to-Noise Ratio (SNR). We denote $R_{n}(t) \in \mathcal{R}$ as the channel state information (CSI) of user $n$ at time $t$. For user $n$, let $p_{k}^{n}$ denote the probability of being state $k$, i.e, $\operatorname{Pr}\left[R_{n}(t)=r_{k}\right]=p_{k}^{n}, n \in \mathcal{N}$, $k \in\{1,2, \ldots, \mathrm{E}\}$ and each channel state is possible with nonzero probability such that $p^{\min }<p_{k}^{n}<p^{\max }, \forall n, i$.

The BS maintains a separate queue for each user, hence, queue size information is available at the scheduler in advance. Packets arrive into users' queues according a stationary arrival process that is independent across users and time slots. Let $A_{n}(t)$ be the amount of data (bits or packets) arriving into the queue of user $n$ at time slot $t$. We denote the arrival rate vector as $\boldsymbol{\lambda}=\left(\lambda_{1}, \lambda_{2}, \ldots, \lambda_{N}\right)$, where $\lambda_{n}=\mathbf{E}\left[A_{n}(t)\right]$.

\section{A. Channel Probing Model}

We assume that at the beginning of a time slot, the BS sends a pilot signal. Based on the quality of the received pilot signal, each user $n$ can determine its current channel state $R_{n}(t)$. However, the CSI of users are not known to the scheduler, hence, a selected number of users (or all users) reports their CSIs to the BS before scheduling a downlink transmission. In practice, reporting the CSI consumes resources like time. We quantify the overhead of obtaining the CSI of a single user in terms of a time fraction of the time slot as in [12]. Specifically, we assume that $\beta$ fraction of the time slot is consumed to obtain CSI from a single user. This time duration may include the time spent for pilot signal transmission, measurement of the signal strength of pilot signal and the transmission of CSI to the base station. We assume that $1-\beta N<1$, i.e., there is always non-zero time duration for data transmission. Let $\mathcal{N}_{p}(t) \subset \mathcal{N}$ be the set of users who report back their CSI. Hence, only $\left(1-\beta N_{p}(t)\right) \times T_{s}$ seconds are available for data transmission where $N_{p}(t)$ is the cardinality of $\mathcal{N}_{p}(t)$ and $T_{s}$ is the duration of the time slot. . When all users report their CSIs to the BS at every time slot, which we call full CSI 
case, then the time available for data transmission is equal to $(1-\beta N) \times T_{s}$.

The transmission to user $n$ cannot be fulfilled unless the CSI is reported. Let $I_{n}(t)$ be an indicator function showing whether user $n$ is scheduled to be transmitted to at time slot $t$ :

$$
I_{n}(t)= \begin{cases}1 & ; \text { if user } n \in \mathcal{N}_{p}(t) \text { and it is scheduled } \\ 0 & ; \text { otherwise }\end{cases}
$$

At most one user can be served at a time slot, i.e., $\sum_{n=1}^{N} I_{n}(t)=1, \forall t$. The amount of data that can be transmitted to user $n$ by the BS when $N_{p}(t)$ users are probed at time $t$ is given by,

$$
D_{n}(t)=\left(1-\beta N_{p}(t)\right) T_{s} R_{n}(t) I_{n}(t) .
$$

We assume that $T_{s}$ is normalized to unit slot length, i.e., $T_{s}=1$ in the rest of the paper. Let $\boldsymbol{Q}(t)=$ $\left(Q_{1}(t), Q_{2}(t), \ldots, Q_{N}(t)\right)$ denote the vector of queue sizes, where $Q_{n}(t)$ is the queue length of user $n$ at time slot $t$.

Definition 1: A queue is strongly stable if

$$
\limsup _{t \rightarrow \infty} \frac{1}{t} \sum_{\tau=0}^{t-1} \mathbf{E}\left[Q_{n}(t)\right]<\infty
$$

Moreover, if every queue in the network is stable then the network is called stable. The dynamics of the queue of user $n$ is given by,

$$
Q_{n}(t+1)=\left[Q_{n}(t)+A_{n}(t)-D_{n}(t)\right]^{+} .
$$

where $[x]^{+}=\max (x, 0)$. We define the weighted rate of user $n$ as follows:

$$
W_{n}(t)=Q_{n}(t) R_{n}(t),
$$

It is well known that the contentional Max-Weight algorithm schedules the user with maximum weighted rate with full CSI [1]. However, acquiring CSI from all users decreases the available time for data transmission, hence, decreases throughput. The next question therefore is does there exist an algorithm that can find the user with maximum weighted rate without probing all users? Next, we show that there exists such an algorithm. Before giving the proposed scheduling algorithm, we first characterize the achievable rate region of the network and give some useful definition.

\section{B. Characterizing Rate Region}

In regarding our model, let us consider a joint scheduling and channel probing policy (possible randomized) $G \in \mathcal{G}$ which is applied to schedule a user, where $\mathcal{G}$ is the set of all possible joint scheduling polices. At every time slot $t, G$ first selects the number of users that is to be probed according to some distribution. We assume that $G$ decides to probe $m$ users with probability $\pi_{m}, m \in\{1,2, \cdots, N\}$. Next, $G$ picks $m$ users and obtain their CSIs according to some another distribution. Let $\alpha_{n}^{m}$ be the probability that $G$ probes user $n$ when $m$ users are probed and $\sum_{n=1}^{N} \alpha_{n}^{m}=1$, for all $m$. Then, $G$ schedules a user among the probed users. Let
$\mathbf{T}_{m}^{G}=\mathbf{E}\left[\mathbf{D}_{m}^{G}(t)\right]$ be the expected throughput achieved with policy $G$ and when $m$ users are probed, where $\mathbf{D}_{m}^{G}(t)=$ $\left[D_{m, 1}^{G}(t), D_{m, 2}^{G}(t), \cdots, D_{m, N}^{G}(t)\right] . D_{m, n}^{G}(t)$ is the throughput achieved by user $n$ with policy $G$ and when $m$ users are probed at time $t . D_{m, n}^{G}(t)$ can be defined similarly as in (2). Hence, the achievable rate region of $G, \Lambda_{G}$, is given by [1]:

$$
\Lambda_{G}=\sum_{m=1}^{N} \pi_{m} \quad \mathcal{C H}\left[\mathbf{T}_{m}^{G} \quad: G \in \mathcal{G}\right]
$$

where $\mathcal{C H}[A]$ as the convex hull of set $A$. The achievable rate region of the network, $\Lambda$, is then defined to be the union of the rate regions for all possible scheduling policies $G$. However, designing such a scheduling algorithm is impossible in practice. Hence, we turn our attention into more practical and online algorithms. Next, we give some definitions for more realistic rate regions.

Definition 2: $\Lambda_{h}$ is the hypothetical rate region where full CSI is available (e.g. by an Oracle) without any channel probing or feedback costs, i.e., $\beta=0$.

Definition 3: $\Lambda_{f}$ is the achievable rate region when probing cost is taken into account and when all users' channels are probed at every time slot according to the feedback model. Note that $\Lambda_{f} \subset \Lambda_{h}$.

\section{SCHEDUling AND DYNAMIC FEEDBACK (SDF) ALGORITHM}

In this section, we propose a joint scheduling and dynamic feedback allocation algorithm that determines the user which has the maximum weighted throughput at each time slot without probing every user.

\section{SDF Algorithm:}

(1) probing decision:

- Step 1: Determine the user which has the maximum queue length,

$$
i^{*} \triangleq \underset{i \in \mathcal{N}}{\operatorname{argmax}}\left\{Q_{i}(t)\right\}
$$

- Step 2: Probe and acquire the CSI of user $i^{*}$. Let $R_{i^{*}}(t)$ be the CSI of user $i^{*}$ at time $t$.

- Step 3: Broadcast the value of $R_{i^{*}}(t)$.

- Step 4: The users which have higher rate than $R_{i^{*}}(t)$ report their CSIs to BS.

Let us define the set $\mathcal{S}_{p}(t)$ as follows:

$$
\mathcal{S}_{p}(t) \triangleq\left\{j: R_{j}(t)>R_{i^{*}}(t)\right\}
$$

(2) scheduling decision:

BS schedules a user which has the maximum weighted throughput according to Max-Weight algorithm [1] as follows;

$$
n^{*}=\underset{n \in \mathcal{N}_{p}(t)}{\operatorname{argmax}}\left\{\left(1-\beta N_{p}(t)\right) W_{n}(t)\right\}
$$

where

$$
\mathcal{N}_{p}(t) \triangleq \mathcal{S}_{p}(t) \cup i^{*}
$$


Intuition: Let us assume that user $n^{*}$ has the maximum weighted rate at a time slot. Given CSI of the user $i^{*}$, the CSI of users with rate lower than $R_{i *}$ need not be collected since their weighted rates are always smaller than that of user $i^{*}$. SDF algorithm is especially efficient when the number of users is large since in that case the number of users with rate lower than that of user $i^{*}$ is large with high probability.

\section{A. Analysis of SDF Algorithm}

Now, we investigate the increase in achievable rate region when SDF algorithm is employed. We establish a bound on the achievable rate region of SDF.

Let $M(t)$ denote the number of users which do not send their CSIs since their channel conditions are worse than the user which has the maximum queue length at time $t$. The number of fractions of time slot consumed for probing with SDF algorithm is determined as follows: first, the BS acquires the CSI of user $i^{*}$ and $\beta$ fraction of time slot is used for probing user $i^{*}$. Then, BS broadcasts the value of CSI of user $i^{*}$. We assume that broadcasting CSI of a user also consumes $\beta$ fraction of time slot. Then, the number of users which have higher rate than $R_{i^{*}}(t)$ is equal to $N-1-M(t)$. Hence, the total number of fractions used for channel probing within SDF algorithm at time $t$ is given as follows:

$$
N_{p}(t)=1+1+N-1-M(t)=N+1-M(t) .
$$

Note that $N_{p}(t)=N$ when all users are probed as in conventional Max Weight algorithm.

We consider the following two functions:

$$
\begin{aligned}
f_{s}(\boldsymbol{Q}(t)) & =\mathbf{E}\left[\sum_{n \in \mathcal{N}_{p}(t)}\left(1-\beta N_{p}(t)\right) W_{n}(t) I_{n}(t) \mid \boldsymbol{Q}(t)\right], \\
f_{m}(\boldsymbol{Q}(t)) & =\mathbf{E}\left[\sum_{n \in \mathcal{N}}(1-\beta N) W_{n}(t) I_{n}(t) \mid \boldsymbol{Q}(t)\right],
\end{aligned}
$$

where the expectation is taken with respect to the randomness of channel variations and scheduling decisions. Given $\boldsymbol{Q}(t)$, both Max-Weight algorithm with full CSI and SDF schedules the same user which has the maximum weighted rate at every time slot. Hence, the value of $W_{n}(t) I_{n}(t)$ is the same for both functions, and the only difference between $f_{s}(\boldsymbol{Q}(t))$ and $f_{m}(\boldsymbol{Q}(t))$ appears in the number of users probed. Let $\Lambda_{s}$ denote the rate region of SDF algorithm. Next,we analyze the performance of SDF algorithm in terms of achievable rate region by using the theorem given in [13].

Theorem 1: [13] If for some $\epsilon>0 \mathrm{SDF}$ algorithm guarantees

$$
f_{s}(\boldsymbol{Q}(t))=(1+\epsilon) f_{m}(\boldsymbol{Q}(t))
$$

for all $\boldsymbol{Q}(t)$, then SDF achieves $1+\epsilon$ fraction of the rate region $\Lambda_{f}$.

Theorem 2: For homogenous channels, SDF algorithm can support $(1+\epsilon)$ fraction of $\Lambda_{f}$, i.e., $\Lambda_{s}=(1+\epsilon) \Lambda_{f}$, where

$$
\epsilon=\frac{\beta(\mathbf{E}[M(t)]-1])}{1-\beta N} .
$$

Proof: By using (7), $f_{s}(\boldsymbol{Q}(t))$ can be rewritten as follows:

$$
\begin{aligned}
& f_{s}(\boldsymbol{Q}(t))= \\
& \mathbf{E}\left[\sum_{n}(1-\beta(N+1-M(t))) W_{n}(t) I_{n}(t) \mid \boldsymbol{Q}(t)\right] \\
& =f_{m}(\boldsymbol{Q}(t))+\mathbf{E}\left[\sum_{n}(\beta M(t)-\beta) W_{n}(t) I_{n}(t) \mid \boldsymbol{Q}(t)\right]
\end{aligned}
$$

Now, we consider the value of $f_{s}(\boldsymbol{Q}(t)) / f_{m}(\boldsymbol{Q}(t))$ such that,

$$
\begin{aligned}
& f_{s}(\boldsymbol{Q}(t)) / f_{m}(\boldsymbol{Q}(t))= \\
& \frac{f_{m}(\boldsymbol{Q}(t))+\mathbf{E}\left[\sum_{n \in \mathcal{N}_{p}(t)}(\beta M(t)-\beta) W_{n}(t) I_{n}(t) \mid \boldsymbol{Q}(t)\right]}{f_{m}(\boldsymbol{Q}(t))} \\
& =1+\frac{\mathbf{E}\left[\sum_{n}(\beta M(t)-\beta) W_{n}(t) I_{n}(t) \mid \boldsymbol{Q}(t)\right]}{f_{m}(\boldsymbol{Q}(t))}
\end{aligned}
$$

where,

$$
\begin{aligned}
& \mathbf{E}\left[\sum_{n \in \mathcal{N}_{p}(t)}(\beta M(t)-\beta) W_{n}(t) I_{n}(t) \mid \boldsymbol{Q}(t)\right]= \\
& {\left[\sum_{m=0}^{N-1}(\beta m-\beta) \mathbf{E}\left[\sum_{n \in \mathcal{N}_{p}(t)} W_{n}(t) I_{n}(t) \mid \boldsymbol{Q}(t), M(t)=m\right]\right]} \\
& \times \operatorname{Pr}[M(t)=m]
\end{aligned}
$$

Note that

$$
\mathbf{E}\left[\sum_{n \in \mathcal{N}_{p}(t)} W_{n}(t) I_{n}(t) \mid \boldsymbol{Q}(t), M(t)=m\right]=\frac{f_{m}(\boldsymbol{Q}(t))}{1-\beta N}
$$

Hence, (11) can be rewritten as follows:

$$
\begin{aligned}
(11) & =\frac{f_{m}(\boldsymbol{Q}(t))}{1-\beta N} \sum_{m=0}^{N-1}[(\beta m-\beta)] \operatorname{Pr}[M(t)=m] \\
& =\frac{\left.\beta f_{m}(\boldsymbol{Q}(t))(\mathbf{E}[M(t)]-1]\right)}{1-\beta N}
\end{aligned}
$$

Thus we have,

$$
f_{s}(\boldsymbol{Q}(t)) / f_{m}(\boldsymbol{Q}(t))=1+\frac{\beta(\mathbf{E}[M(t)]-1])}{1-\beta N}
$$

Hence, SDF algorithm can support $(1+\epsilon)$ fraction of $\Lambda_{f}$ where $\epsilon=\frac{\beta(\mathbf{E}[M(t)]-1])}{1-\beta N}$. This completes the proof.

Note that according to Theorem 2, the performance of SDF algorithm in terms of rate region depends on only $\mathbf{E}[M(t)]$. Clearly, if $\mathbf{E}[M(t)]>1$, then $\epsilon>0$. In other words, we can increase the rate region. In addition, $\epsilon$ is given in general in the sense that it does not depend on channel characteristics. However, to find explicit bound on $\epsilon$ one needs to determine $\mathbf{E}[M(t)]$. Next, we determine the value of $\mathbf{E}[M(t)]$ when channels are homogenous. 


\section{B. Performance of SDF with Homogenous Channels}

In this section, we determine $\mathbf{E}[M(t)]$ by considering homogenous channels and find the exact value of $\epsilon$. According to homogeneous channel model, each user has the same channel distribution, i.e.,

$$
p_{k}^{n}=p_{k}, \forall n .
$$

We begin with a Lemma that shows how to determine the value of $\mathbf{E}[M(t)]$ when channels are homogenous.

Lemma 1: When channels are homogenous, $\mathbf{E}[M(t)]$ is given as follows:

$$
\begin{aligned}
& \mathbf{E}[M(t)]= \\
& {\left[p_{1}+p_{2}\left(\sum_{k=2}^{L} p_{k}\right)+p_{3}\left(\sum_{k=3}^{L} p_{k}\right)+\ldots+p_{L}^{2}\right](N-1)}
\end{aligned}
$$

Proof: Due to the space limitation, we omit the proof. Details of the proof are available in our online technical report [14].

Next, we investigate the case when the channels are homogenous and uniformly distributed such that $p_{k}=\frac{1}{L}$ for all $k$.

Lemma 2: When channels are homogenous and uniformly distributed, $\mathbf{E}[M(t)]$ is given as follows:

$$
\mathbf{E}[M(t)]=\left[\frac{1}{2}+\frac{1}{2 L}\right](N-1)
$$

Proof: If all channels are uniformly distributed, then $p_{k}=$ $\frac{1}{L}$. In that case, by using (13) $\mathbf{E}[M(t)]$ is given by,

$$
\begin{aligned}
\mathbf{E}[M(t)] & =(N-1) \times \\
& {\left[\frac{1}{L}+\frac{1}{L}\left(\sum_{k=2}^{L} \frac{1}{L}\right)+\frac{1}{L}\left(\sum_{k=3}^{L} \frac{1}{L}\right)+\ldots+\frac{1}{L^{2}}\right] }
\end{aligned}
$$

Thus, we have,

$$
\begin{aligned}
\mathbf{E}[M(t)] & =(N-1) \times \\
& {\left[\frac{1}{L}+\frac{1}{L^{2}}(L-1)+\frac{1}{L^{2}}(L-2)+\ldots+\frac{1}{L^{2}}\right] }
\end{aligned}
$$

When we arrange the above terms, we obtain

$$
\begin{aligned}
\mathbf{E}[M(t)] & =\left[\frac{1}{L}+\frac{L(L-1)}{2 L^{2}}\right](N-1) \\
& =\left[\frac{1}{2}+\frac{1}{2 L}\right](N-1)
\end{aligned}
$$

Theorem 3: When channels are homogenous and if $N>3$ we guarantee to expend the rate region, i.e., $\epsilon>0$ and the amount of increase in rate region is equal to $\epsilon$ given in (8).

Proof: The proof of the theorem can be done in two parts. In the first part, we show that $\mathbf{E}[M(t)]$ is minimum when channels are uniform. In the second part of the proof, we show that if $N>3$, then $\epsilon>0$ when channels are uniform. Due to the space limitation, we omit the proof. Details of the proof are available in our online technical report [14].

\section{NumericAl RESUlts}

In our simulations, we model a single cell CDMA downlink transmission utilizing high data rate (HDR) [4]. The base station serves 20 users and keeps a separate queue for each user. Time is slotted with length $T_{s}=5 \mathrm{~ms}$. Packets arrive at each slot according to Poisson distribution for each users with mean $\lambda_{n}$. The size of a packet is set to 128 bytes which corresponds to the size of an HDR packet. Each channel has 5 possible states with rates as given in Table I.

TABLE I: Possible Physical Rates

\begin{tabular}{|l|l|l|l|l|l|}
\hline Rates & $r_{1}$ & $r_{2}$ & $r_{3}$ & $r_{4}$ & $r_{5}$ \\
\hline $\mathrm{kb} / \mathrm{s}$ & 1843.2 & 1228.8 & 614.4 & 307.2 & 76.8 \\
\hline
\end{tabular}

\section{A. Homogenous Channels}

First, we evaluate the performance of SDF algorithm when channels are homogenous and non-uniform distributions.

1) Non-Uniform Channels: Here, we investigate the performance of SDF algorithm when channels are identical but the channel state distributions are not uniform. In this case, the channel state distribution is given in Table II:

TABLE II: Channel state distribution

\begin{tabular}{|l|l|l|l|l|l|}
\hline & $p_{1}$ & $p_{2}$ & $p_{3}$ & $p_{4}$ & $p_{5}$ \\
\hline probabilities & 0.1 & 0.1 & 0.2 & 0.3 & 0.3 \\
\hline
\end{tabular}

Figure 2 depicts the average total queue sizes in terms of packets vs. the overall arrival rate for $\beta=0.02$ and $\beta=0.03$. We also depict the performance the algorithm (Cha) given in [12]. Clearly, the maximum supportable arrival rate is achieved in hypothetical case, i.e., $\beta=0$. When $\beta=0.02$, the minimum supportable rate is achieved with Cha algorithm. This is due to the fact that Cha algorithm does not guarantee to find the user with maximum weighted throughput even if it does not probe every user at each time slot. However, when $\beta=0.03$, then channel probing decreases throughput significantly and Cha outperforms full CSI case. It is easy to see that for both values of $\beta$, SDF outperforms Cha and achieves higher maximum supportable rate. This is due to that fact that SDF algorithm guarantees to schedule the user with maximum weighted throughput at every time slot by probing less than $50 \%$ of users. Specifically, we observe that SDF algorithm only probes less than $50 \%$ of all channels, i.e., $\mathbf{E}[M(t)]=11.7$.

2) Non-Stationary Channels: Next, we demonstrate the performance of SDF algorithm over time-correlated and nonstationary channels. The channel between the BS and each user is modeled as a correlated Rayleigh fading according to Jakes' model with different Doppler frequencies varying randomly between $5 \mathrm{~Hz}$ and $15 \mathrm{~Hz}$. We set $\mathrm{BW}=1.25 \mathrm{MHz}$ and SNR $=10 \mathrm{~dB}$. Let $H_{n}(t)$ denote CSI of user $n$ at time 

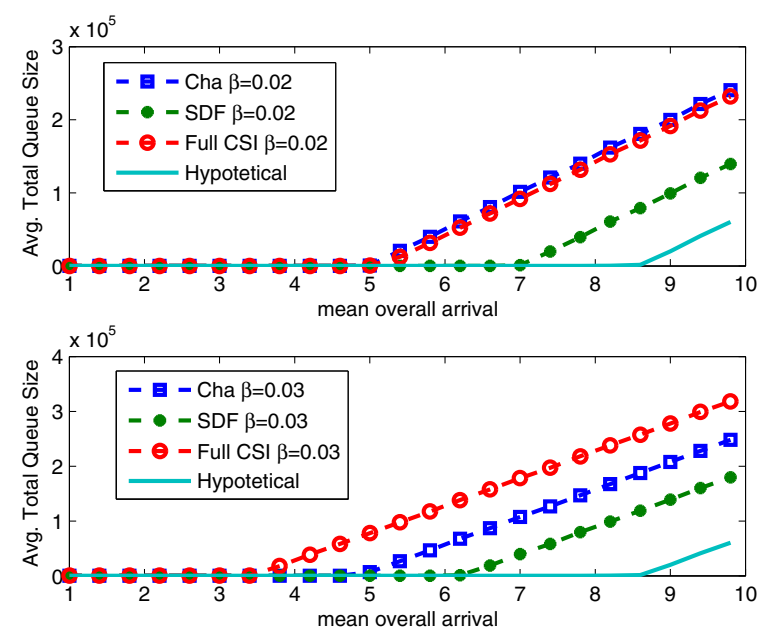

Fig. 2: Performance of SDF algorithm with Homogenous and Non-Uniform channels.

slot $t . H_{n}(t)$ is a random process which does not have a stationary probability distribution, i.e, the mean of the channel gain changes over time. Let $h_{n}(t)$ represent the realization of $H_{n}(t)$ at time $t, n \in\{1,2, \ldots, N\}$. Then, the maximum number of bits that user $n$ can transmit is given as,

$$
R_{n}(t)=T_{s}\left(1-\beta N_{p}(t)\right) \mathrm{BW} \log _{2}\left(1+\mathrm{SNR} \times h_{n}(t)\right)
$$

where $B W$ is the bandwidth of a channel. Similar to the previous scenario, Figure 3 depicts the average total queue sizes in terms of packets vs. the overall arrival rate when the channels are non-stationary and $\beta=0.02$. The maximum and the minimum arrival rates while keeping the queues stabile are achieved in hypothetical and full CSI cases, respectively. As the overall arrival rate exceeds 14 packets/slot queue sizes suddenly increase with full CSI Max-Weight and the network becomes unstable. However, SDF improves over full CSI MaxWeight by supporting the overall arrival rate of up to 16 packets/slot. In other words, SDF algorithm is able to sustain $14 \%$ more traffic than the full CSI Max-Weight algorithm. Thus, SDF appears to stabilize a larger range of arrival rates.

\section{CONCLUSION}

In this paper, we have considered the joint scheduling and channel probing problem in a single channel wireless downlink network. We have developed a low complex and dynamic feedback algorithm named SDF. We have shown that SDF algorithm can support $1+\epsilon$ fraction of full achievable rate region. Then, we have proved the sufficient condition for $\epsilon>0$. For homogenous channels, the simulation results demonstrated that by probing less than $50 \%$ of users, our algorithm expands the rate region. In this work, the analytical and simulation results are given by assuming homogenous channels and a single channel wireless network. As a future work, we will investigate the performance of SDF by considering both heterogenous channels and multichannel wireless system, i.e., OFDM networks. Another future research direction is to study the case where probing error is taken into account.

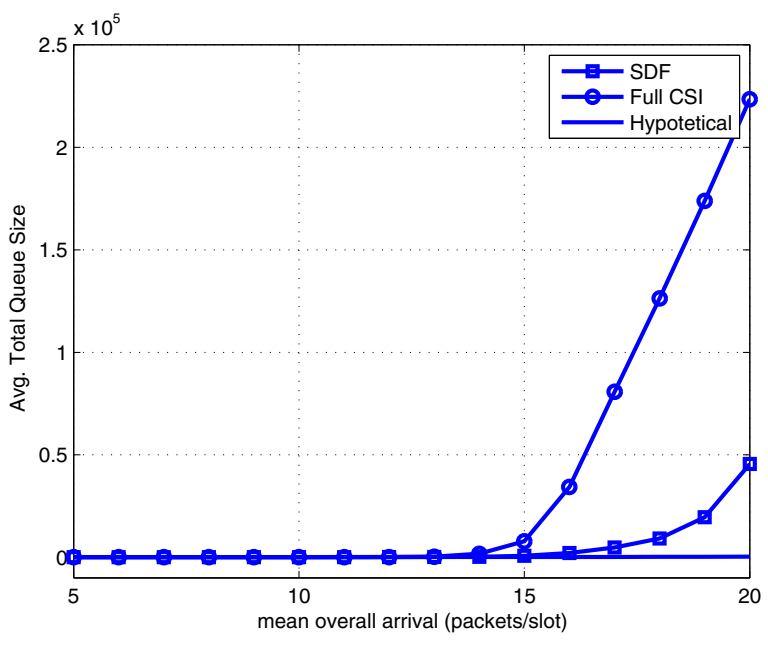

Fig. 3: Performance of SDF algorithm over non-stationary channels.

\section{REFERENCES}

[1] L. Tassiulas and A. Ephremides, "Stability properties of constrained queueing systems and scheduling policies for maximum throughput in multihop radio networks," IEEE Trans. Autom. Control, vol. 37, no. 12, pp. 1936 - 1948, Dec. 1992.

[2] X. Liu, E. K. P. Chong, and N. B. Shroff, "A framework for opportunistic scheduling in wireless networks," Comput. Netw., vol. 41, 2003.

[3] M. J. Neely, "Energy optimal control for time varying wireless networks," IEEE Trans. Inf. Theory, vol. 52, no. 7, pp. 2915 - 2934, 2006.

[4] P. Bender, P. Black, M. Grob, R. Padovani, N. Sindhushyana, and S. Viterbi, "Cdma/hdr: a bandwidth efficient high speed wireless data service for nomadic users," IEEE Commun. Mag., vol. 38, no. 7, pp. $70-77,2000$.

[5] A. Gopalan, C. Caramanis, and S. Shakkottai, "On wireless scheduling with partial channel-state information," in Proc. of the 45th Allerton Conference, 2007.

[6] M. Ouyang and L. Ying, "On optimal feedback allocation in multichannel wireless downlinks," in Mobihoc, 2011.

[7] C.-P. Li and M. J. Neely, "Energy-optimal scheduling with dynamic channel acquisition in wireless downlinks," IEEE Trans. Mobile Comput., vol. 9, no. 4, p. 527 539, Apr. 2010.

[8] M. J. Neely, "Max weight learning algorithms with application to scheduling in unknown environments," in arXiv:0902.0630v1, Feb. 2009.

[9] W. Ouyang, S. Murugesan, A. Eryilmaz, and N. B. Shroff, "Scheduling with rate adaptation under incomplete knowledge of channel/estimator statistics," in 48th Allerton Conference on Communication, Control, and Computing, 2010.

[10] _ "Exploiting channel memory for joint estimation and scheduling in downlink networks," in INFOCOM, 2011.

[11] C. Tekin and M. Liu, "Approximately optimal adaptive learning in opportunistic spectrum access," in INCOFOM, 2012.

[12] P. Chaporkar, A. Proutiere, H. Asnani, and A. Karandikar, "Scheduling with limited information in wireless systems," in Mobihoc, 2009.

[13] A. Eryilmaz, R. Srikant, and J. Perkins, "Stable scheduling policies for fading wireless channels," IEEE/ACM Trans. Netw., vol. 13, pp. 411424, 2005.

[14] M. Karaca, Y. Sarikaya, O. Ercetin, T. Alpcan, and H. Boche, "Throughput optimal scheduling with dynamic channel feedback," http://arxiv.org/abs/1203.5362. 